\title{
Les occurrences de [ç] dans le parler de Languidic
} (breton haut-vannetais)

The occurrences of $[c ̧]$ in the Languidic dialect (Upper Vannetais Breton)

\section{Maxime-Morvan Crahé}

\section{(2) OpenEdition}

\section{Journals}

Édition électronique

URL : http://journals.openedition.org//bl/435

DOI : $10.4000 / \mathrm{lbl} .435$

ISSN : 2727-9383

Éditeur

Université de Bretagne Occidentale - UBO

\section{Édition imprimée}

Date de publication : 1 mars 2016

Pagination : 125-133

ISBN : 979-10-92331-24-0

ISSN : 1270-2412

Référence électronique

Maxime-Morvan Crahé, "Les occurrences de [ç] dans le parler de Languidic (breton haut-vannetais) », La Bretagne Linguistique [En ligne], 20 | 2016, mis en ligne le 01 mai 2020, consulté le 01 octobre 2020 URL : http://journals.openedition.org/lbl/435 ; DOI : https://doi.org/10.4000/lbl.435

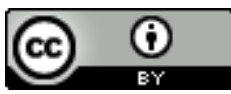

La Bretagne Linguistique est mise à disposition selon les termes de la Licence Creative Commons Attribution 4.0 International. 
Maxime-Morvan CRAHÉ*

\title{
Les occurrences de [ç] dans le parler de Languidic (breton haut-vannetais)
}

\begin{abstract}
D ès les premiers échanges avec des locuteurs traditionnels de breton languidicien, un son consonantique attire l'attention : "Le coucher du soleil ? C'est [ərçø়hçâwl] en breton » me répondit ma principale informatrice. En effet, la consonne fricative palatale sourde apparaît fréquemment, alors qu'elle est inexistante dans une bonne partie du domaine bretonnant notamment au nord-ouest; et bien qu'elle soit notée dans plusieurs transcriptions phonétiques des monographies portant sur des parlers bretons du sud-est, elle n'est pas toujours présentée dans leur système consonantique. Voyons donc, à travers ses occurrences, quelle est la pertinence de ce phonème dans le parler de Languidic.
\end{abstract}

\section{[ç] allophone palatal de [h]}

D'un point de vue phonologique, [ç] apparait comme un allophone de [h]. L'articulation [h] est palatalisée au contact de [j]. Ainsi, /hj/ est réalisé [ç]. Ce phénomène principalement constaté dans le sudest de la Basse-Bretagne (dialecte vannetais et les parlers de la frange cornouaillaise limitrophe) peut être rapproché, dans une moindre mesure, des cas d'allophonie $\mathrm{du} / \mathrm{x} /$, de l'allemand $<\mathrm{ch}>$, ou encore $\mathrm{du}$ grec $\langle\chi\rangle$. Bon nombre d'exemples de [ç] relevés à Languidic se retrouvent dans les autres parlers vannetais. Certains d'entre eux, présentés plus bas, laissent supposer l'apparition de [h] ou [j] dans leurs réalisations courantes.

* Professeur certifié de breton. Docteur en celtique, CRBC, UHB Rennes 2/ubl. 


\begin{tabular}{|c|c|c|c|}
\hline $\begin{array}{c}\text { Breton } \\
\text { peurunvan }\end{array}$ & $\begin{array}{c}\text { Breton } \\
\text { languidicien }\end{array}$ & Français & Remarques \\
\hline heol & [çawl] & soleil & /hjawl/ \\
\hline go(i)añv ${ }^{2}$ & [gu'jã:] [gu'çã: ] & hiver & $\begin{array}{l}\text { Ces deux réalisations cohabitent, } \\
\text { parfois dans un même idiolecte. }\end{array}$ \\
\hline merien & [kərç'õ: ] & mouches & $\begin{array}{l}\text { - Apparition supposée, comme } \\
\text { dans d'autres parlers vannetais } \\
\text { d'un }[\mathrm{h}] \text { après la liquide, ici }[\mathrm{r}]^{3} \text {. } \\
\text { - Modification de la liquide }[1]>[\mathrm{r}]\end{array}$ \\
\hline $\begin{array}{c}\text { lizher } \\
\text { mezherenn }\end{array}$ & 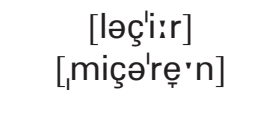 & $\begin{array}{l}\text { lettre } \\
\text { lange }\end{array}$ & $\begin{array}{l}\text { - Apparition supposée de [j] de } \\
\text { transition entre les deux timbres } \\
\text { vocaliques }\end{array}$ \\
\hline
\end{tabular}

Cette apparition, purement phonétique, de [ç] dans les mots ne contenant qu'un seul morphème est rare. En revanche, celle-ci est plus fréquente en dérivation lors de l'ajout de suffixes commençant par [j], à des morphèmes, principalement des noms, terminés par $[\mathrm{h}]$.

\begin{tabular}{|c|c|c|}
\hline \multicolumn{3}{|c|}{ [jœ'y] morphème du pluriel $<$ ioù $>($ variante de $<$ où $>)$} \\
\hline Breton peurunvan & Breton languidicien & Français \\
\hline mouezh / mouezhioù & 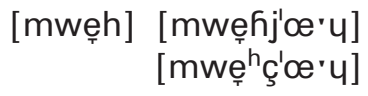 & voix (sing.) / voix (pluriel) \\
\hline \multicolumn{3}{|c|}{ [jat] morphème exprimant le contenu $<$ iad $>($ variante de $<$ ad $>)$} \\
\hline devezh / devezhiad & 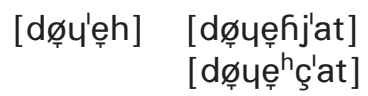 & journée / journées \\
\hline
\end{tabular}

\section{Remarques :}

- Le $/ \mathrm{h} / \mathrm{a}$, dans le parler, une large zone d'articulation et ses réalisations varient selon l'environnement phonétique, mais aussi selon l'intonation ou le locuteur, comme dans ces exemples.

- Lorsque [ç] apparaît dans ce cas, une fricative gutturale est maintenue avant celui-ci.

1. Geriadur Brezhoneg An Here, Le Relecq-Kerhuon, Embannadurioù An Here, 2001.

2. Le [j] réalisé dans plusieurs parlers bretons vannetais dans ce mot, noté ici (i) n'apparaît pas en breton peurunvan.

3. Peut-être par mimétisme avec l'exemple précédent. 
- Les exemples de noms terminés par [h] recevant ces suffixes, notamment [jœ'y], sont nombreux.

\begin{tabular}{|c|c|c|}
\hline \multicolumn{3}{|c|}{ [jẹt] morphème du pluriel $<$ ied $>($ variante de $<\mathrm{ed}>)$} \\
\hline Breton peurunvan & Breton languidicien & Français \\
\hline merc'h/merc'h(i)ed & [mẹh] . [mir'jẹt] [mir'çe̦t] & fille / filles \\
\hline
\end{tabular}

\section{Remarque :}

- Dans cet exemple unique, l'apparition de [j] introduisant ce morphème du pluriel comme dans les autres parlers du sud de Basse-Bretagne ${ }^{4}$, peut être considérée comme une variante du morphème du pluriel $<\mathrm{ed}>$. Cette variante n'est pas notée dans la graphie peurunvan.

\begin{tabular}{|c|c|c|}
\hline \multicolumn{3}{|c|}{ [ji:r] morphème du pluriel <ier $>$} \\
\hline Breton peurunvan & Breton languidicien & Français \\
\hline bazh / bizhier & [bah] [bəç'i:r] & bâton / bâtons \\
\hline kloc'h / kleier & {$\left[\mathrm{kləç}^{\prime} \mathrm{i}: r\right]^{5}$} & cloche / cloches \\
\hline garzh / girzhier & [gah] [gərçli:r] & talus boisé / talus boisés \\
\hline forc'h / ferchier & [fơ⿱日十)] [fərç'i:r] & fourche / fourches \\
\hline
\end{tabular}

\section{Remarques:}

- Les exemples sont assez rares, compte tenu du faible rendement de ce morphème du pluriel. Comme dans les autres dialectes, le timbre de la voyelle originelle est modifié dans cette dérivation. Cette voyelle courte est dans ce cas dépourvue d'accent tonique dans le parler, aussi son timbre est neutralisé, et s'articule [ə].

- Nous pouvons aussi ajouter à cette liste d'exemples, le pluriel de [ti: ] «maison», rare nom recevant le morphème pluriel <ji:r> ne finissant pas par/h/, en [tijiir] / [təj'irr] mais aussi fréquemment [tiçiir] / [təç'iir], sans doute par mimétisme.

- Certains locuteurs articulent ces pluriels sans fricative, comme par exemple [sø̛ji:r] « sacs ».

4. Carte $\mathrm{n}^{\circ} 446$ dans Pierre Le Roux, Atlas linguistique de la Basse-Bretagne, Rennes, Plihon et Hommay, 1924-63, dorénavant ALBB.

5. Notons d'ailleurs un rare cas d'homophonie selon les locuteurs, et le tempo [ərləç'irr] [ər $\left.{ }^{\mathrm{h}}\right)$ ləç'i:r] la lettre/ les cloches. 


\begin{tabular}{|c|c|c|}
\hline \multicolumn{3}{|c|}{ [ẹ:s] morphème du féminin $<\mathrm{ez}>$} \\
\hline Breton peurunvan & Breton languidicien & Français \\
\hline $\begin{array}{c}\text { nizez } \\
\text { gwerc'hez }\end{array}$ & $\begin{array}{c}\text { [niç'è:s] } \\
\text { [dzyẹrçe̦:s] }\end{array}$ & $\begin{array}{c}\text { nièce } \\
\text { Vierge }\end{array}$ \\
\hline
\end{tabular}

\section{Remarques :}

- $\quad$ Nous pouvons supposer l'apparition de [j] de transition entre les deux timbres vocaliques de ces exemples uniques.

- La graphie <nizez> présentée ici peut être vue comme une formation du féminin fréquente, c'est à dire l'ajout du morphème du féminin $<$ ez $>$ à un nom masculin singulier, dans ce cas précis $<$ niz $>$ « neveu ». Mais en observant les réalisations courantes de ce mot dans tout le domaine bretonnant ${ }^{6}$, et son étymologie, nous pouvons supposer l'ajout de ce morphème du féminin, par redondance, à un nom alors déjà féminin nith, « nièce » en vieux breton, que l'on retrouve dans la graphie nizhez ${ }^{7}$, ainsi que dans sa réalisation languidicienne présentée ici.

\section{[c]] spirantisation de [t]]}

D'un point de vue morphophonologique [h] peut être le résultat d'une mutation consonantique par spirantisation $\mathrm{du} / \mathrm{k} /$.

En ce qui concerne les consonnes vélaires, rappelons que le phénomène de palatalisation des de $/ \mathrm{k} / \mathrm{et} / \mathrm{g} /$ est bien connu dans le sud-est du domaine bretonnant, et qu'il a généralement évolué jusqu'à des réalisations

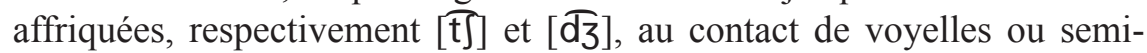
voyelles antérieures et du timbre neutralisé [ə] des voyelles inaccentuées, mais qu'il n'est pas systématique dans le parler en question.

Ainsi, [ç] apparaît dans ce parler comme la spirantisation du [t]] initial, uniquement lorsqu'il est suivi de la voyelle [Øָ] souvent neutralisée [ə] à un tempo courant. Observons donc la mutation des phonèmes initiaux suivants après [ur], l'article indéfini singulier, provoquant ici la spirantisation de ces noms masculins singuliers :

6. ALBB carte $n^{\circ} 496$.

7. Francis FAVEREAU, Dictionnaire du breton contemporain - bilingue, Morlaix, Skol Vreizh, 1993. 
- kazh / ur c'hazh

[kah]

[urh'ah]

chat/ un chat

- ki / ur c'hi

[t]i: ]

[urhi: ]

chien / un chien

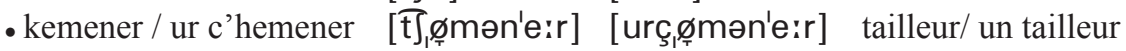

Voici les autres mutateurs de cette spirantisation, présentés avec

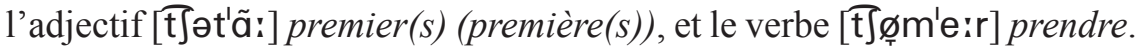

\begin{tabular}{|c|c|c|}
\hline Mutateur & Classe grammaticale & Exemple \\
\hline$[\varnothing r]$ & Article défini & $\begin{array}{l}\text { [ərçət'ã: ] } \\
\text { le premier }\end{array}$ \\
\hline [mø] & $\begin{array}{l}\text { Déterminant possessif et pronom possessif } \\
\text { objet de la } 1{ }^{\text {re }} \text { personne du singulier. }\end{array}$ & 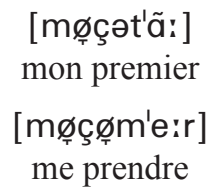 \\
\hline [i] & $\begin{array}{l}\text { Déterminant possessif et pronom possessif } \\
\text { objet de la } 3^{\mathrm{e}} \text { personne du singulier féminin, } \\
\text { des temps simples. }\end{array}$ & $\begin{array}{c}\text { [içət'ã:] } \\
\text { son premier } \\
\text { [içø̛'te:r] } \\
\text { la prendre }\end{array}$ \\
\hline$[\mathrm{ur}]^{8}$ & $\begin{array}{l}\text { Déterminant possessif et pronom possessif } \\
\text { objet de la1 }{ }^{\text {re }} \text { personne du pluriel. }\end{array}$ & $\begin{array}{c}\text { [urçət'ã:] } \\
\text { notre premier } \\
\text { [urçøฺm'e:r] } \\
\text { nous prendre }\end{array}$ \\
\hline$[\mathrm{u}]$ & $\begin{array}{l}\text { Déterminant possessif et pronom possessif } \\
\text { objet de la } 3^{\mathrm{e}} \text { personne du pluriel, des temps } \\
\text { simples. }\end{array}$ & $\begin{array}{l}\text { [uçət'ãa: } \\
\text { leur premier } \\
\text { [uçø̛m'e:r] } \\
\text { les prendre }\end{array}$ \\
\hline [Øָr] & $\begin{array}{l}\text { Pronom possessif objet de la } 3^{\mathrm{e}} \text { personne du } \\
\text { singulier masculin, des temps simples. }\end{array}$ & $\begin{array}{l}\text { [Øָrçøฺm'e:r] } \\
\text { le prendre }\end{array}$ \\
\hline
\end{tabular}

Les occurrences de cette mutation sont malgré tout assez faibles puisque peu de noms ou adjectifs présentent [ $\overparen{\mathrm{t}} \not \varnothing]$ à l'initiale.

8. [nø̄] a parfois été relevé pour cette même personne. Il est lui aussi mutateur de la spirantisation. 


\section{[ç] provection de [j]}

Toujours d'un point de vue morphophonologique [ç] doit être considéré comme le corrélat non voisé de $/ \mathrm{j} /$, et apparaît donc comme le résultat de la mutation consonantique par provection $\mathrm{du} / \mathrm{j} /[\mathrm{j}]$ initial.

Voici les phonèmes initiaux voisés du breton languidicien concernés par la provection :

\begin{tabular}{|c|c|c|c|c|c|c|c|c|}
\hline & Labiales & Dentales & Labio-vélaires & Vélaires & $\begin{array}{l}\text { Chuin- } \\
\text { tantes }\end{array}$ & Palatale & \multicolumn{2}{|c|}{ Liquides } \\
\hline $\begin{array}{l}\text { Occlusives } \\
\text { nasales }\end{array}$ & $/ \mathrm{m} /$ & $/ \mathrm{n} /$ & & & & & & \\
\hline \multirow{2}{*}{$\begin{array}{l}\text { Occlusives } \\
\text { orales }\end{array}$} & \multirow[t]{2}{*}{$/ \mathrm{b} /$} & \multirow[t]{2}{*}{$/ d /$} & $\mid \overparen{g w} /$ & $/ g /$ & & & & \\
\hline & & & {$[d \widetilde{34}] \quad[\overparen{g w}]$} & \begin{tabular}{l|l}
{$[\bar{d}]$} & {$[g]$}
\end{tabular} & & & & \\
\hline \multirow[t]{2}{*}{ Spirantes } & \multirow[t]{2}{*}{$/ \mathrm{v} /$} & \multirow[t]{2}{*}{$|z|$} & $/ \mathrm{w} /$ & & \multirow[t]{2}{*}{$|3|$} & \multirow[t]{2}{*}{$/ \mathrm{j} /$} & \multirow[t]{2}{*}{ /l/ } & \multirow[t]{2}{*}{$|r|$} \\
\hline & & & {$[w]$} & & & & & \\
\hline
\end{tabular}

Voyons leurs articulations après mutation par provection, notée phonologiquement avec le signe diacritique du dévoisement $/ \mathrm{l}$ :

\begin{tabular}{|c|c|c|c|c|c|c|c|c|c|}
\hline & Labiales & Dentales & Labio-vélaires & \multicolumn{2}{|c|}{ Vélaires } & Chuin- & Palatale & \multicolumn{2}{|c|}{ Liquides } \\
\hline $\begin{array}{l}\text { Occlusives } \\
\text { nasales }\end{array}$ & $\begin{array}{c}/ \mathrm{m} / \\
{\left[{ }^{\mathrm{h}} \mathrm{m}\right]}\end{array}$ & $\begin{array}{l}/ \mathrm{n} / \\
{\left[{ }^{\mathrm{h}} \mathrm{n}\right]}\end{array}$ & & & & & & & \\
\hline \multirow{2}{*}{$\begin{array}{l}\text { Occlusives } \\
\text { orales }\end{array}$} & \multirow{2}{*}{$\begin{array}{l}\text { /b/ } \\
{[p]}\end{array}$} & \multirow{2}{*}{$\begin{array}{l}/ \mathrm{d} / \\
{[\mathrm{t}]}\end{array}$} & $|\overparen{g w}|$ & \multicolumn{2}{|c|}{$\lg /$} & & & & \\
\hline & & & {$[\mathrm{t}[\mathrm{u}] \quad[\overline{\mathrm{kw}}]$} & $[\widehat{t}]]$ & [k] & & & & \\
\hline \multirow[t]{2}{*}{ Spirantes } & \multirow[t]{2}{*}{$/ \mathrm{v} /$} & \multirow[t]{2}{*}{$|z|$} & $/ \mathrm{w} /$ & & & \multirow{2}{*}{$\begin{array}{l}13.1 \\
{[S]}\end{array}$} & \multirow{2}{*}{$\begin{array}{l}/ \mathrm{j}_{\circ} / \\
{[\mathrm{c}]}\end{array}$} & \multirow{2}{*}{$\begin{array}{l}/ / / \\
{\left[{ }^{h} I\right]}\end{array}$} & \multirow{2}{*}{$\begin{array}{l}/ \mathrm{r} / \\
{\left[{ }^{\mathrm{h}} \mathrm{r}\right]}\end{array}$} \\
\hline & & & {$\left[{ }^{\mathrm{h}} \mathrm{u}\right] \quad\left[{ }^{\mathrm{h}} \mathrm{w}\right]$} & & & & & & \\
\hline
\end{tabular}

- Notons que l'aspiration notée [h] peut souvent être accompagnée d'un léger dévoisement des phonèmes consonantiques initiaux.

- Ajoutons que les voyelles mutent elles aussi par provection, et sont réalisées précédées de $\left.{ }^{\mathrm{h}}\right]$, et pourraient être ajoutées au système, notées $/ \varnothing /$, devenant $/ \varnothing / /\left[{ }^{\mathrm{h}} \emptyset\right]$ par provection.

Le dévoisement à l'initiale dans un contexte de provection s'applique traditionnellement à l'ensemble du système consonantique, mais tend à disparaître pour quelques articulations (liquides, nasales, semi-consonnes) chez certains locuteurs, notamment les plus jeunes. Ceci est peut-être à 
rapprocher du phénomène général d'amuïssement du [h], en particulier à l'initial, connu dans de nombreux parlers haut-vannetais.

Néanmoins la provection de $/ \mathrm{j} /$, notée ci dessus $/ \mathrm{j}_{\mathrm{o}} /$ est toujours réalisée [c] après les pronoms possessifs de la $2^{\mathrm{e}}$ personne du pluriel [u], et de la $2^{\mathrm{e}} \mathrm{du}$ singulier féminin [i] puisqu'elle sert à les distinguer des pronoms possessifs homophones $[\mathrm{u}]\left(3^{\mathrm{e}}\right.$ pers. pluriel) et [i] ( $3^{\mathrm{e}}$ pers. masculin singulier) qui eux ne provoquent pas la provection.

\begin{tabular}{|c|c|c|}
\hline [ja:r] poule & $\begin{array}{c}\text { [iç'a:r] } \\
\text { sa poule (à elle) } \\
\text { [uç'a:r] } \\
\text { votre/ta poule }\end{array}$ & $\begin{array}{c}\text { [i'ja:r] } \\
\text { sa poule (à lui) } \\
\text { [u'ja:r] } \\
\text { leur poule }\end{array}$ \\
\hline [jõ:t] oncle & $\begin{array}{c}\text { [iç'õ:t] } \\
\text { son oncle (à elle) } \\
\text { [uç'õ:t] } \\
\text { votre/ton oncle }\end{array}$ & $\begin{array}{c}\text { [i'jõ:t] } \\
\text { son oncle (à lui) } \\
\text { [u'jõ:t] } \\
\text { leur oncle }\end{array}$ \\
\hline $\begin{array}{l}\text { [jəvã: } \widehat{\text { tj'i:s] }} \\
\text { jeunesse }\end{array}$ & 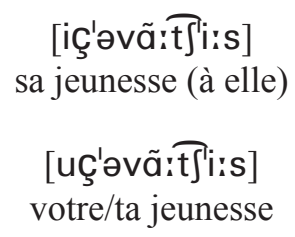 & 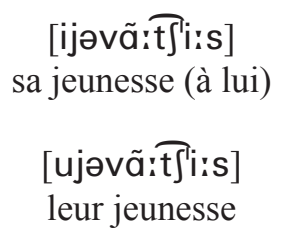 \\
\hline
\end{tabular}

Rappelons que le tutoiement est inconnu à Languidic. Ainsi, la deuxième personne du pluriel est l'unique forme exprimant la deuxième personne, tant au singulier qu'au pluriel. Cette habitude langagière fait que la provection est très fréquente.

\section{Conclusion}

Nous constatons que [ç] apparaît lors de phénomènes phonétiques et morphophonologiques uniquement.

L'articulation [c]], bien qu'elle soit la plus courante dans les exemples présentés plus haut, n'est pas la seule. Elle est une des nombreuses réalisations de $/ \mathrm{h} /$ et apparait dans le parler comme son allophone palatal, en variante combinatoire avec [h], ou résultant de la spirantisation des

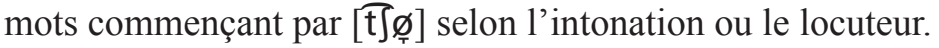


En revanche, [ç] devient pertinent, dans un contexte morphophonologique, en tant que provection de [j].

Les nombreuses occurrences de [c]], relevées auprès d'une vingtaine de locuteurs traditionnels, nous invitent à penser que le breton de Languidic se trouve dans une région, voire un terroir où ce son s'articule le plus fréquemment dans le paysage linguistique de Basse-Bretagne.

\section{Remarque annexe : la mutation $[\mathrm{g}]>[\mathrm{j}]$}

Il y a dans le parler un autre cas de consonne occlusive vélaire mutant en fricative palatale. La mutation par lénition $\mathrm{du} / \mathrm{g} / \mathrm{g}]$ initial propose plusieurs réalisations ${ }^{9}$ dans le parler, dont [j] à considérer ici comme une fricative palatale sonore. Cette réalisation semble s'appliquer aux noms singuliers féminins ${ }^{10}$, uniquement après les mutateurs : [ur] article indéfini singulier, [Øָr] article défini, et [diu] adjectif numéral cardinal 2 au féminin et [i] pronom possessif de la $3^{\mathrm{e}}$ personne du singulier masculin.

\begin{tabular}{|c|c|}
\hline [go:r] & chèvre \\
[ø్'jo:r] & la chèvre \\
[diujo:r] & deux chèvres \\
[i'jo:r] & sa chèvre (à lui) \\
\hline [ga:r] $^{1 \text { 'gai }}$ & jambe \\
[ør'ja:r] & la jambe \\
[diuja:r] $^{11}$ & deux jambes \\
[i'ja:r] & sa jambe (à lui) \\
\hline
\end{tabular}

\section{Bibliographie}

CHEVEAU Loïc, Approche phonologique, morphologique et syntaxique du breton du Grand Lorient (bas-vannetais), thèse UHB Rennes 2.

CRAHÉ Maxime-Morvan, Le breton de Languidic : étude phonétique, morphologique et syntaxique d'un sous-dialecte vannetais, thèse de doctorat, UHB Rennes 2, 2013.

9. La lénition du groupe des vélaires sonores initiales (incluant les palatales et/ou les labialisées) ne semble pas systématique et reste encore à être décrite plus précisément. .

10. Sauf les noms commençant par [gu] ou le cluster labialisé [gw].

11. Homophonie dans le parler : la jambe / la poule, <ar c'har / ar yar>. 
EVENOU Erwan, Studi fonologel brezhoneg Lanijen (Kanton ar Faoued, Kernev), thèse de doctorat, Université de Rennes II, 1987.

FAVEREAU Francis, Dictionnaire du breton contemporain - bilingue, Morlaix, Skol Vreizh, 1993

HuMPHREYS Humphrey, Phonologie et morphosyntaxe du parler breton de Bothoa, Emgelo Breiz, 1995.

LE PIPEC Erwan, Le breton de Malguénac, Ploemeur, Hor Yezh, 2000.

LE RouX Pierre, Atlas linguistique de la Basse-Bretagne (ALBB), Rennes, Plihon et Hommay, 6 fascicules 1927 [Reprint : Brest, éd. Armoricaines, 1977].

MC KENNA Malachy, « The Breton of Guéméné-sur-Scorff (bas-vannetais) », Zeitschrift für celtische Philologie, 1976.

Rolland Pascal, Atlas linguistique de la région de Pontivy, Brest, Ar Skol Vrezoneg, 1994. 